\title{
Gota tofácea: ¿indisciplina o desconocimiento?
}

M. Metola Gómez, J. J . Dones Carvajal*, Mạ. A. Camacho Pastor

Especialista en Medicina Familiar y Comunitaria. Centro de Salud Mạ M ontessori.

Leganés. Área 9 de Madrid. *Especialista en M edicina Interna.

Clínica Mondoa. Madrid

Tophic gout: indiscipline or ignorance?

\section{RESUMEN}

La gota es una enfermedad con una etiopatoge nia clara, diagnóstico preciso y tratamiento eficaz. A pesar de ello, un porcentaje no despreciable de pacientes desarrollan gota tofácea crónica. Su pre sencia se correlaciona con el tiempo de evolución de la enfermedad y con el grado de elevación de los niveles de ácido úrico. Realizando desde nues tro ámbito un diagnóstico precoz y un tratamiento adecuado conseguiremos disminuir los ataques agudos, prevenir la artropatía crónica y reducir las complicaciones extraarticulares de los pacientes con hiperuricemia.

Palabras clave: Tofos. Artritis gotosa. Gota to fácea. Atención Primaria.

\begin{abstract}
Gout is a disease with clear etiopathogeny, pre cise diagnosis and effective treatment. Neverthe less, a significant percentage of patients develops tophic chronic gout. Its presence is correlated with the time of evolution and the degree of elevation of the uric acid level. By performing, from the scope of the primary Health Care, an early diagnosis and suitable treatment, we will be able to reduce acute attacks, prevent chronic arthropathy and minimize extraarticular complications in patients with hype ruricaemia.
\end{abstract}

Key words: Tophus. Gouty arthritis. Tophic Gout. Primary Health Care.

\section{INTRODUCCIÓN}

La hiperuricemia es una enfermedad metabólica frecuente, con una prevalencia que oscila entre un 2 y un $13,2 \%$ en la población adulta ${ }^{1,2}$. Su repercusión asistencial en el marco de la Atención Primaria no viene determinada únicamente por su incidencia, sino por sus manifestaciones clínicas y su consideración como factor de riesgo cardiovascular. Las tres situaciones clínicas más importantes que pueden presentarse en nuestra consulta son la hiperuricemia asintomática, aquella asociada a li- tiasis renal y la relacionada con gota. Esta última se desarrolla en el $1 \%$ de los pacientes hiperuricémicos. Las manifestaciones clínicas son debidas al depósito de cristales de urato monosódico (UMS) en las articulaciones, zonas periarticulares y otras estructuras. Los tofos corresponden a agregados de cristales de UMS en la piel y el tejido celular subcutáneo, rodeados de una reacción inflamatoria de células gigantes. En casos dudosos de gota, el hallazgo de los mismos puede decidir el diagnóstico, aunque lo habitual es que se trate de gota con historia típica donde el tofo es otro dato clínico más.

Aceptación: 17-01-02 
Como en el caso que nos ocupa, es característica del paciente gotoso su clásica indisciplina en cuanto a la adhesión al tratamiento ${ }^{2}$, pero también la falta de habilidades, tanto diagnósticas como terapéuticas, que en el manejo de la gota pueden determinar un curso tórpido de la enfermedad en nuestros pacientes.

\section{OBSERVACIÓN CLÍNICA}

Varón de 54 años, mecánico de profesión, sin antecedentes familiares de interés. No presentaba antecedentes quirúrgicos, alergias a medicamentos ni hábitos tóxicos, ni hipertensión arterial, ni diabetes mellitus, ni hipercolesterolemia conocidas. Refería el diagnóstico de gota a los 38 años de edad. Había presentado, durante años, ataques de monoartritis agudas en distintas localizaciones (pies, tobillos y manos). En la mayoría de los episodios no había consultado, porque se autolimitaban en pocos días sin dejar secuelas funcionales. No seguía tratamiento dietético ni farmacológico. Consultó por dolor cólico abdominal, localizado en flanco derecho y con irradiación a genitales, expulsión de litiasis, disuria y polaquiuria. Se encontraba afebril. El resto de anamnesis por órganos y aparatos era negativa excepto podalgia alternante y algias ocasionales en rodillas y muñecas, con cierta limitación funcional progresiva para el desarrollo manual de su trabajo. En la exploración física destacaba la presencia de múltiples tumoraciones de consistencia firme a nivel de metatarsos (Fig. 1), muñecas y metacarpofalángicas (Fig. 2), ambas bolsas olecranianas y rodillas (Fig. 3), sin signos inflamatorios en la piel circundante salvo enrojecimiento local. Presentaba un índice de masa corporal (IMC) de $32 \%$ y, a la palpación abdominal, dolor en flanco y fosa ilíaca derechos con puñopercusión renal derecha positiva. El resto de

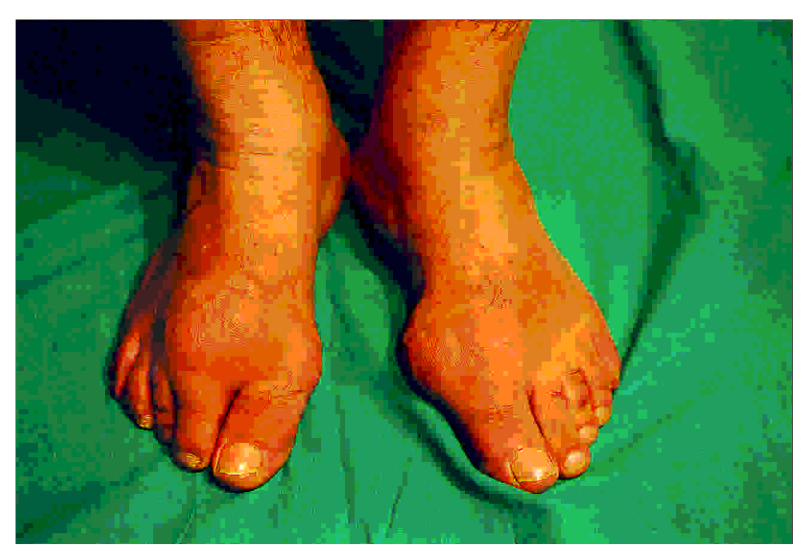

Figura 1

Tofos en articulaciones metatarsofalángicas.

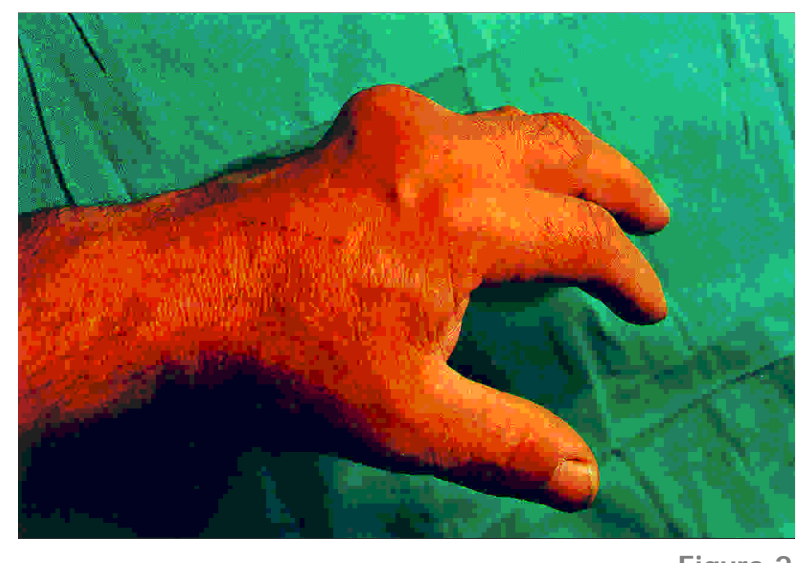

Figura 2

Tofos en muñecas y metacarpofalángicas.

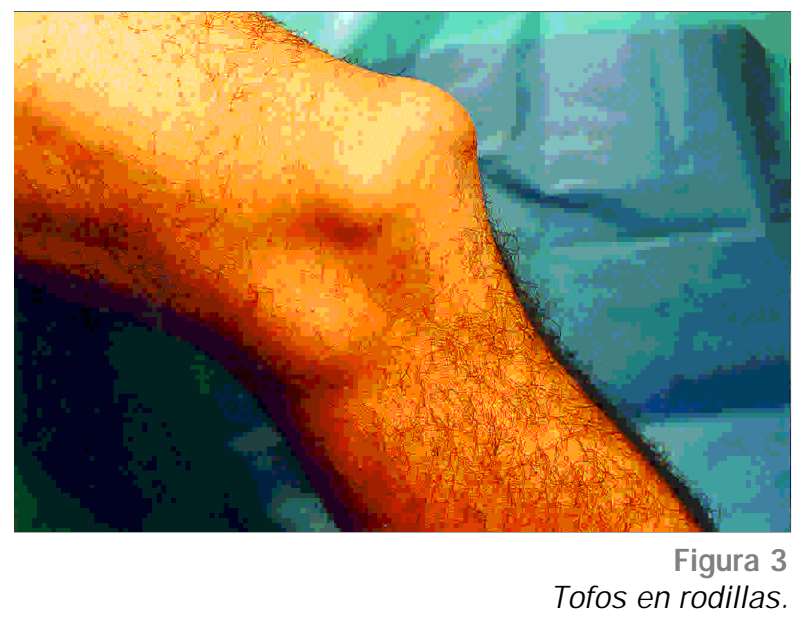

la exploración era normal. Se realizó analítica general con hemograma normal, bioquímica normal (incluyendo función renal y ácido úrico de 5,9 $\mathrm{mg} / \mathrm{dl}$ ). Sistemático y sedimento de orina con hematuria microscópica y presencia de bacterias, urocultivo negativo, uricosuria de 24 horas de $580 \mathrm{mg}$, análisis bioquímico de la litiasis correspondiente a oxalato cálcico. Se solicitó radiografía (RX) de abdomen simple con imagen sugerente de litiasis renal derecha, urografía intravenosa con presencia de litiasis pielocalicial derecha no complicada, RX de manos, codos, rodillas, tobillos y pies con signos sugestivos de gota tofácea: lesiones osteolíticas a nivel de distintas articulaciones metatarsofalángicas de ambos pies, estiloides cubital y huesos del carpo de la mano derecha, cabeza de primer a tercer metacarpiano de mano izquierda, coracoides de ambos cúbitos y rótula derecha. Aumento de densidad y volumen de partes blandas periarticulares, calcificaciones múltiples en el interior de los tofos correspondientes, deformidades en los dedos de ambos pies, reacción esclerosa a nivel rotuliano y disminución de 
interlínea articular en varias articulaciones metatarsofalángicas. El paciente contaba con el diagnóstico previo de artritis por depósito de cristales de UMS tras análisis de líquido articular en uno de sus primeros episodios de monoartritis en pie izquierdo, por lo que no se repitió. El último episodio de artritis había tenido lugar hacía aproximadamente 5 meses por lo que se instauró tratamiento profiláctico con colchicina a dosis de 1 $\mathrm{mg} /$ día y posteriormente tratamiento hipouricemiante con alopurinol a dosis de $100 \mathrm{mg} /$ día. Se recomendó, asimismo, una dieta baja en purinas e ingesta abundante de agua.

\section{DISCUSIÓN}

El ácido úrico es el producto final del metabolismo de las purinas, que debe eliminarse a través de la orina. Los valores considerados normales son de $7 \mathrm{mg} / \mathrm{dl}$ en el varón y $6 \mathrm{mg} / \mathrm{dl}$ en la mujer ${ }^{3}$. Por encima de éstos, el ácido úrico puede precipitar en forma de cristales de UMS desencadenando las distintas formas clínicas de la enfermedad. La hiperuricemia viene determinada fundamentalmente por alteraciones del metabolismo de las purinas presentes desde el nacimiento, y que se potencian en personas con ingesta elevada de las mismas y de alcohol. En otros casos, el aumento se determina por un exceso de producción (enfermedades mielo y linfoproliferativas, policitemia vera, anemias hemolíticas, neoplasias sólidas, psoriasis extensas, obesidad, consumo excesivo de alcohol, etc.) o por disminución de la eliminación (insuficiencia renal, riñón poliquístico, nefropatía por plomo, consumo excesivo de alcohol, fármacos, etc.), casos que corresponderían a formas secundarias de hiperuricemia. La gota es una enfermedad causada por el depósito de ácido úrico en las articulaciones con una respuesta inflamatoria ante la presencia de pequeños cristales de UMS ${ }^{2}$.

Existen tres presentaciones clínicas de la gota: el ataque agudo de artritis (generalmente monoarticular), el periodo intercrisis y la gota tofácea.

La primera es la más frecuente, sobre todo en la primera articulación metatarsofalángica, aunque también es frecuente la afectación de rodilla, tobi1lo, pie medio, muñecas,... El primer ataque suele ser brusco, con dolor intenso, edema y eritema marcados. Suele ser autolimitado y, si bien la clínica sugiere fuertemente el diagnóstico de gota, el definitivo se constata con la presencia de cristales de UMS en el líquido sinovial. Estos aparecen como cristales en forma de aguja con birrefringencia negativa en el microscopio de luz polarizada. La clínica de artritis monoarticular también se puede dar en otras patologías con las que debemos establecer un diagnóstico diferencial como la artropatía por depósitos de cristales de pirofosfato cálcico (condrocalcinosis), infecciones o espondiloartropatía como la enfermedad de Reiter o la psoriasis s $^{3,4}$.

La gota intercrítica es el periodo que acontece entre episodios agudos. Puede ser asintomática o cursar con dolor o deformidad articular.

Por último, la artritis gotosa crónica o gota tofácea, se caracteriza por la presencia de tofos que, en ocasiones, son el origen de erosiones del cartílago y del hueso subcondral ${ }^{5}$. Se trata de tumoraciones de tamaño variable y corresponden a depósitos de UMS rodeados de células mononucleares con reacción a cuerpo extraño. Se forman en las articulaciones, alrededor de las mismas o en zonas alejadas. Las localizaciones más frecuentes son el hélix, bolsas olecranianas, manos, muñecas, rodillas, tendón Aquíleo y pies $^{1,6}$. La piel que los recubre puede tener una coloración blanquecina, blanco-amarillenta, enrojecida como en el caso descrito o tener un aspecto normal. La consistencia puede ser blanda y fluctuante como una colección purulenta, o dura, como en el caso de los tofos antiguos o en localizaciones determinadas. En las bolsas serosas pueden palparse, a veces, nódulos duros dentro del material blando. Ocasionalmente se ulceran y dejan salir un material blanquecino y pastoso. Pueden también infectarse, pero no es lo habitual ${ }^{7}$. Por lo general, los tofos tienden a crecer lentamente mientras el paciente mantiene niveles elevados de ácido úrico; por ello -salvo excepciones- son una manifestación tardía de la gota ${ }^{8}$. Su presencia se correlaciona con el tiempo de evolución de la enfermedad, con un tratamiento inadecuado de la misma o con la severidad de la hiperuricemia ${ }^{9}$. El caso presentado sería un claro ejemplo, dado que el diagnóstico se estableció hace 16 años pero el paciente reconocía no haber tenido nunca en cuenta las recomendaciones en cuanto a dieta, tratamiento farmacológico e indicaciones de seguimiento médico.

Radiológicamente durante el ataque agudo, salvo el aumento de volumen de partes blandas, no suele haber alteraciones. En la artropatía gotosa crónica nos podemos encontrar depósitos uráticos intraóseos, erosiones en la superficie articular, disminución de la interlínea articular, reacción esclerosa secundaria, etc. Los tofos suelen ser radiotransparentes, sólo visibles radiológicamente si son muy voluminosos o están calcificados.

Los niveles de ácido úrico suelen ser elevados pero pueden existir manifestaciones clínicas, incluso artritis aguda, con niveles normales en ese momento.

El tratamiento de la enfermedad por depósito de cristales de UMS es distinto que el del ataque agudo. Al tratar la enfermedad se intentan prevenir futuros ataques, disolver los tofos y evitar nuevos depósitos de cristales ${ }^{10}$. Para ello es preciso realizar una dieta baja en purinas, forzar la diuresis y alcali- 
nizar la orina, y fármacos en los casos necesarios. En ocasiones, debido a la gran deformidad y alteraciones estructurales de las articulaciones es necesario un tratamiento quirúrgico reparador ${ }^{11}$. La dieta tiene baja eficacia y un alto porcentaje de incumplimientos; no obstante, se hará hincapié en la restricción de alcohol y determinados alimentos (vísceras, embutidos, mariscos). El objetivo será disminuir los niveles séricos de ácido úrico por debajo de $5 \mathrm{mg} / \mathrm{dl}$. Para tal efecto los hipouricemiantes son los medicamentos de elección ${ }^{3}$. El tratamiento debe ser de por vida, hecho que debemos explicar al paciente, así como que los medicamentos no están exentos de toxicidad y que aun con tratamiento puede haber un ataque agudo. Un error en el que aún se incurre es la suspensión del tratamiento tras el ataque agudo o cuando se lleva utilizando durante un tiempo prolongado ${ }^{2}$. Antes de iniciar el tratamiento con reductores de uratos, el paciente no debe presentar ningún signo inflamatorio y está indicada la profilaxis previa con colchicina $(1 \mathrm{mg} /$ día $)$ para evitar ataques agudos ante la movilización de los depósitos intraarticulares de los cristales de urato. El fármaco de elección es el alopurinol, utilizado en dosis de 100 a 400 mg/día. Los fármacos uricosúricos (benzobromarona) estarían contraindicados en nuestro paciente por la existencia de nefrolitiasis. Asimismo, no pueden utilizarse en caso de insuficiencia renal.
En resumen, en el marco de la Atención Primaria, es fundamental realizar un diagnóstico precoz, establecer la sospecha clínica y realizar las pruebas complementarias que permitan confirmarlo, controlar al paciente ya diagnosticado vigilando la aparición de posibles complicaciones extraarticulares y monitorizar adecuadamente los niveles de ácido úrico y el tratamiento prescrito. También nos corresponde conseguir que el paciente hiperuricémico reciba una información precisa respecto a su enfermedad, evolución, cronicidad de los cuidados y necesidad de adhesión al tratamiento. Mejorando, pues, nuestras habilidades en el manejo práctico de esta entidad y mejorando la comunicación con el paciente, el objetivo de que casos como el que nos ocupa sean excepcionales estará más cercano.

\section{CORRESPONDENCIA:}

Montserrat Metola Gómez

Centro de Salud $\mathrm{M}^{\mathrm{a}}$ Montessori

Avda. de Portugal $n^{\circ} 2$

28916 Leganés. Madrid

Tfno.: 916804411

\section{Bibliografía}

1. Wortman L. Gota y otros trastornos del metabolismo de las purinas. En: Harrison. Principios de Medicina Interna. 14 ed. Madrid: Mc Graw Hill Interamericana de España, 1998. p. $2455-63$.

2. Ballina FJ, Jirout Casillas F, Martín Lascuevas P. Las enfermedades microcristalinas. En: Ballina FJ, Rodríguez A, Arribas JM. Los Reumatismos Inflamatorios. Manual para la Atención Primaria. $1^{a}$ ed. Madrid: Ediciones Ergon, S.A, 1999. p. 183-97.

3. Rull M. Artropatías por cristales. Revista de Investigación Clínica 2000; 52: 451-60.

4. Rothschild BM, Mas AT. Dactylitis: Implications for clinical practice. Semin Arthritis Rheum 1998; 28: 41.

5. Hollinworth P, Scott TJ, Burry HC. Nonarticular gout: Hyperuricemia and tophus formation without gouty arthritis. Semin Arthritis Rheum 1984; 13: 360-8.

6. Fernández M, Juanola X. Problemas osteoarticulares. En:
Martín Zurro A. Atención Primaria. $4^{\mathrm{a}}$ ed. Madrid: Harcourt Brace de España, 1999. p. 1136-38.

7. Roig D. Reumatología en la consulta diaria. $2^{\mathrm{a}}$ ed. Barcelona: Espaxs, 1997. p. 157-66.

8. Wernick R, Winkler C, Campbell S. Thopi as the initial manifestation of gout. Report of six cases and review of the literature. Arch Intern Med 1992; 152: 873.

9. Pascual E. Hiperuricemia y gota. En: Pascual E. Manual de enfermedades reumáticas de la Sociedad Española de Reumatología (SER). Madrid: Mosby/Doyma Libros S.A, 1996. p. 637-45.

10. Pérez F, Calabozo M, Ugalde J, Herrero AM. Artropatías microcristalinas I. Hiperuricemia y gota. Medicine (Barc) 2001; 34: 1765-80.

11. Piza-Kaltzer H, Komurcu F, Reining-Festa A. Surgical therapy of pronounced gout tophi both hands. Case report. Hand Mik Plast Chir 1997; 29: 96-100. 